\title{
Efficacy and safety of cross-linked hyaluronic acid single injection on osteoarthritis of the knee: a post-marketing Phase IV study
}

This article was published in the following Dove Press journal:

Drug Design, Development and Therapy

8 April 2015

Number of times this article has been viewed

\author{
Khaldoon Bashaireh' \\ Ziad Naser ${ }^{2}$ \\ Khaled Al Hawadya ${ }^{2}$ \\ Sorour Sorour ${ }^{2}$ \\ Rami Nabeel Al-Khateeb ${ }^{3}$ \\ 'Department of Orthopedics Surgery, \\ King Abdullah University Hospital, \\ Jordan University of Science and \\ Technology, Irbid, Jordan; ${ }^{2}$ Private \\ Clinic, ${ }^{3}$ Elaf Medical Supplies Company, \\ Amman, Jordan
}

Purpose: The primary objective of this study was to evaluate the efficacy, safety, and duration of action of viscosupplementation with Crespine ${ }^{\circledR}$ Gel over a 9-month period.

Materials and methods: The study was a post-marketing Phase IV study. A total of 109 participants with osteoarthritis of the knee (grades 1-4) in the tibio-femoral compartment were recruited in Jordan. Data were collected from each participant during the baseline visit. Each participant received Crespine ${ }^{\circledR} \mathrm{Gel}$ injection, and follow-up visits took place at 3 months, 6 months, and 9 months post-injection.

Main outcome measure(s): An assessment of participants by phone was conducted at 1 month, 2 months, 4 months, 5 months, 7 months, and 8 months post-injection. Western Ontario and McMaster Universities Arthritis Index questionnaires were completed during each visit. A 72-hour visit questionnaire was used to assess the safety of the injection. Statistical analysis included a two-sided 95\% confidence interval for the difference between pain scores across visits, and the percent change from baseline was calculated.

Main results: The full analysis included 84 participants who gave their informed consent and finished the necessary baseline and follow-up visits needed to assess efficacy and safety. Peak improvement was noted at 5 months post-injection, when pain and physical performance scores had decreased to 2.60 and 9.90 , respectively, and the stiffness score was 0.33 . The peak improvement in stiffness was noted at 8 months post-injection, when the stiffness score had decreased to 0.32. Significant improvements were still apparent at 9 months post-injection, when the pain score was 3.36 , the stiffness score was 0.42 , and the physical performance score was 11.5. All side effects were local and transient, and included pain, swelling, and redness of the knee. Most side effects were treated.

Conclusion: Hyaluronan should be encouraged as an alternative or adjunct treatment to oral analgesics to reduce their required doses, and delay potential future surgical intervention.

Keywords: osteoarthritis, hyaluronic acid, intra-articular injection, Crespine ${ }^{\circledR} \mathrm{Gel}$

\section{Introduction \\ Background}

Osteoarthritis $(\mathrm{OA})$ is a chronic degenerative disease of the joints characterized by articular pain, cartilage degeneration, and the loss of normal joint function. ${ }^{1}$ It is the most common form of arthritis, and is often debilitating. ${ }^{1,2}$ The prevalence of OA increases with age, and more than $60 \%$ of individuals over 60 years old experience some cartilage abnormality in a major joint. ${ }^{2}$ The knee is a common site of OA, and subjects with knee OA exhibit a characteristic pattern of decrements in function, generally concerning mobility, transfer from seated or supine position to standing, and activities of daily living (ADLs) involving the lower extremities. Knee OA may 
or may not be symptomatic, and the associated radiographs are either normal or abnormal. ${ }^{3}$

To date, the management strategies for OA have been directed at symptoms, primarily pain. ${ }^{2}$ Clinical management of $\mathrm{OA}$ is directed at reducing pain, maintaining or improving joint mobility, and limiting functional impairment. The American College of Rheumatology (ACR) guidelines suggest initial nonpharmacological therapy (involving patient education, physical therapy, weight loss, exercise, unloaders, or assistive devices), followed by various oral medications for alleviating pain, as the first-line therapy for OA. For patients with defined mechanical abnormalities or severe OA, surgical intervention, including arthroscopy and joint replacement, may be indicated. ${ }^{4,5}$

Pharmacological therapy for OA initially includes simple analgesics, such as acetaminophen and over-thecounter nonsteroidal anti-inflammatory drugs (NSAIDs), and may also consist of topical agents such as capsaicin cream. However, oral NSAIDs have been associated with serious side effects, including severe adverse effects on the gastrointestinal system, and their long-term use is not advised. ${ }^{4,6}$ In patients who fail to obtain adequate pain relief from the aforementioned conservative treatments, selective cyclooxygenase-2 (COX-2) inhibitors or prescription NSAIDs with gastro-protective agents are recommended. Some studies have suggested that COX-2 inhibitors may reduce some of the serious adverse events associated with NSAIDs, but this remains an area of controversy, particularly since the worldwide withdrawal of rofecoxib in September 2004. Treatment with short-term opioids or intra-articular steroids may be considered for acute exacerbations of pain caused by OA flares, but long-term use of these agents can be detrimental. ${ }^{4}$ In such cases, intra-articular corticosteroids are widely used because they provide rapid pain relief, but their benefit appears to be short-lived. ${ }^{6}$ Recently, the ACR guidelines have been updated to include recommendations for the use of intra-articular hyaluronan (HA). ${ }^{4}$

HA is a carbohydrate polymer that is widely distributed in the cartilage, synovial fluid, skin, and aqueous humor, where it plays an important role in maintaining normal joint function by providing support and lubrication, as well as by regulating biochemical processes. ${ }^{2,6,7}$ The viscoelastic properties of HA are responsible for some of the protective functions of synovial fluid, including shock absorption, traumatic energy dissipation, protective coating of the articular surface, and lubrication. HA has a variety of effects on cells in vitro; it reduces leukocyte count and interleukin-1-induced prostaglandin synthesis, stabilizes lysosomal membranes, inhibits phagocytosis and chemotaxis of inflammatory cells, and removes free radicals and other reactive oxygen species. ${ }^{6}$

In OA of the knee, synovial fluid elastoviscosity, synovial fluid HA concentration, and the molecular weight of HA are reduced. ${ }^{4,6} \mathrm{HA}$ concentration and molecular weight are reduced because HA is depolymerized and cleared at higher rates in patients with OA than in normal individuals. ${ }^{8}$ Rapid clearance of HA impairs shock absorption, lubrication, and protection of the joint by the synovial fluid. ${ }^{5}$ Intra-articular HA treatment is aimed at restoring the normal viscoelastic properties of synovial fluid to relieve the signs and symptoms of OA. ${ }^{6}$

Numerous clinical trials have documented the efficacy of single courses of HA for pain relief and improvement of joint function. ${ }^{4}$ Injected HA is cleared from the joint in less than one day, but the benefits of a single treatment are claimed to last several months. ${ }^{6}$ Different mechanisms have been proposed to explain this prolonged action of HA, including stimulation of de novo HA synovial synthesis, suppression of cartilage matrix degeneration, and suppression of inflammatory responses to interleukin- $1 .^{8}$

\section{Objective}

The primary objective of this study was to evaluate the efficacy, safety, and duration of action of viscosupplementation with Crespine ${ }^{\circledR}$ Gel over a 9-month period (end point of the study). The secondary objective was to evaluate the quality of life and time-dependent changes in the Western Ontario and McMaster Universities Arthritis Index (WOMAC) score following the treatment of patients. A paired $t$-test for primary and secondary variables was used to demonstrate treatment efficacy, where the primary variable was the WOMAC pain score, and the secondary variables included WOMAC stiffness, functional, emotional, and social performance scales.

\section{Hypothesis}

Null hypothesis: there is no difference in WOMAC pain score, WOMAC stiffness score, or functional, emotional, or social scales from baseline (pretreatment) to 9 months after viscosupplementation with Crespine ${ }^{\circledR}$ Gel (post-treatment).

\section{Design}

This was a prospective, nonrandomized, unblinded, Phase IV, multicenter, and post-marketing study with consecutive participant enrollment.

\section{Participant flow}

A total of 109 participants gave their consent to participate in the study, while only 84 participants gave their consent 
and completed all visits. Participants who missed visits were excluded from the analysis.

\section{Materials and methods}

Four physicians from different regions in Jordan participated in this study. All investigators used identical case report forms to record data. The case report form included each participant's demographics, physical examination results, inclusion/exclusion criteria, medical history, and dated X-ray imaging. Participant screening took place 2 weeks prior to the baseline visit.

The Ethics Committee and Health Authority approved the study protocol and all participants gave their informed written consent prior to their inclusion in the study. The study involved participants with knee OA, in whom the investigators had decided to start treatment with intra-articular Crespine $^{\circledR}$ Gel injection. Standing plain radiographs were taken with the knee extended. Rotation of the foot was adjusted to keep the second metatarsal bone parallel to the $\mathrm{X}$-ray beam. X-ray radiographs were evaluated for the presence of OA as defined by the Kellgren-Lawrence (KL) scale depicted in the Atlas of Standard Radiographs of Arthritis $(0=$ normal, $1=$ doubtful OA, $2=$ minimal OA, $3=$ moderate $\mathrm{OA}$, and $4=$ severe $\mathrm{OA}$ ). The KL scale is based on the degree of osteophyte formation, joint space narrowing, sclerosis, and joint deformity. All efficacy analyses were performed on the intent-to-treat population, which was defined as those participants who received the single injection of $\mathrm{HA}$, and for whom post-baseline data from follow-up visits at 3 months, 6 months, and 9 months post-treatment were available.

The safety profile of HA viscosupplementation has been well established over its 20 years of clinical use. In fact, no viscosupplement product has been withdrawn because of safety concerns. Intra-articular HA is generally well tolerated and has a low incidence (approximately $1 \%-4 \%$ per injection) of local adverse events. The most common adverse event associated with HA viscosupplementation is a local reaction at the injection site, consisting of mild pain, swelling, or effusion, as well as warmth, redness, or both warmth and redness. Such injection site reactions are usually mild and self-limiting, resolve within 1-3 days, and generally respond to NSAIDs and local modalities. Other mild adverse effects reported after HA viscosupplementation include post-injection itching, headaches, and calf pain. Furthermore, the incidence of adverse events associated with HA viscosupplementation is similar to that observed with other intra-articular procedures and is significantly related to the injection technique used; a medial HA injection for a partially bent knee was associated with an adverse event rate of $5.2 \%$, in comparison with a rate of $1.5 \%$ for straight lateral injections. Interestingly, lateral injection has also been shown to be a more accurate intraarticular administration method than injections into a flexed knee using conventional arthroscopic portal approaches. ${ }^{9}$

The safety assessment was based on reports of adverse events collected between the signing of the informed consent form and the completion of the final follow-up visit for each participant. The frequency of local adverse events, defined as the occurrence of an effusion (evidence from clinical examination or arthrocentesis) or a flare (hot, painful, swollen knee occurring within 48 hours of injection of the study preparation), of corticosteroid injections or treatment interpretations due to local adverse events, and of serious adverse events (adverse events leading to serious disability, hospital admission, life-threatening events, or death) were included in the protocol to be reported.

\section{Participants}

Participants were 40+ years old and diagnosed with OA of the knee in the tibio-femoral compartment. Exclusion criteria included concomitant inflammatory joint disease, concomitant psoriatic arthritis, previous intra-articular fracture of the knee, allergy to any substance related to the study medication (including disinfectants and adhesives), renal impairments, or metastatic tumors.

\section{Intervention(s)}

All participants in the study received intra-articular injections $(2 \mathrm{~mL})$ of Crespine ${ }^{\circledR}$ Gel, a cross-linked HA product.

\section{Outcome measure(s)}

The WOMAC Likert 3.0 is a self-administered, diseasespecific, health-related quality of life instrument that asks the participants to answer questions concerning the study knee. The WOMAC Likert 3.0 produces one aggregate total score and scores for three subscales: pain, stiffness, and physical functioning. A higher score for each subscale corresponds to a worse condition. The pain subscale includes five questions concerning the degree of pain experienced with certain positions and activities (eg, sitting or lying), with a subscale score ranging from 0 to 20 . The function subscale includes 17 questions involving the degree of difficulty experienced while completing activities (eg, descending stairs), with a subscale score ranging from 0 to 68 . The stiffness subscale includes two questions on stiffness severity (ie, after first awaking and later in the day), with the subscale score ranging from 0 to 8 . For every question in the WOMAC, participants rate their 
pain, stiffness, or function using five ordinal responses: none, mild, moderate, severe, and extreme. ${ }^{10}$ The WOMAC scale was designed to measure the dysfunction and pain associated with OA of the lower extremities by assessing 17 functional ADLs, five pain related activities, and two stiffness categories. The WOMAC pain category covers pain associated with walking and stair climbing, as well as nocturnal, rest, and weightbearing pain, while the stiffness category includes morning stiffness and stiffness occurring later in the day. The physical function category includes descending stairs, ascending stairs, rising from sitting, standing, bending to floor, walking on a flat surface, getting in or out of a car, going shopping, putting on socks, rising from bed, taking off socks, lying in bed, sitting, getting on or off of the toilet, heavy domestic duties, and light domestic duties. Performance on social functions and the status of emotional function were also included in the 17 functional activities, in addition to five functions describing the symptoms. The 17 questions were scored as slight (mild), moderate, severe, extreme, or none to determine the daily functional activity status of each participant. Higher WOMAC scores indicated worse pain, stiffness, and functional limitations.

\section{Sample size}

The study sample size was not calculated statistically, but the study was estimated to have sufficient power with a $10 \%$ dropout rate, and to provide sufficient evidence that HA is an effective treatment for knee OA.

\section{Statistical methods}

The primary statistical analysis of efficacy was performed on the full analysis set (FAS). The percent change from baseline and a two-sided $95 \%$ confidence interval (CI) for the difference between pain scores between visits were calculated, and the same procedure was performed for the secondary variables (stiffness and physical function). Quantitative variables were described by the mean, standard deviation, and range. The efficacy of the treatment was examined by a paired $t$-test for both primary and secondary variables. All analyses were performed using the SAS System Release 9.1.3 Software Program (SAS Institute, Cary, NC, USA). Three SAS procedures were added for use in visit evaluations: 1) SAS Proc for means of equal and unequal variances, 2) SAS Proc for histograms (G-plots), and 3) SAS Proc for Box Plots.

\section{Results}

\section{Participant flow}

The FAS consisted of all participants who gave their informed consent and completed the study. The main analysis (the analysis of primary and secondary variables), baseline assessments (eg, demographic data, patient characteristics, and history of cardiovascular diseases), and analysis of survey data were based on participant flow (Figure 1). The study included 109 participants who gave their consent to participate in the study, while only 84 participants gave their consent and completed all visits. Participants with missing visits were excluded from the analysis.

\section{Baseline data}

All participants were 40 years of age or older, and the mean age of the participants was 55.83 years. Most participants were males of Middle Eastern origin. Participant demographics and baseline characteristics are listed in Table 1.

The medical history of the enrolled participants is shown in Figure 2. Many participants had other muscular/skeletal diseases (30.68\%). Cardiovascular disease was also prevalent among the selected participants, as highlighted by the fact that participants with cardiovascular disease either suffered from diabetes or hypertension.

\section{Statistical analysis of the efficacy of Crespine ${ }^{\circledR} \mathrm{Gel}$}

The knee pain of each participant was assessed on their initial visit and 3 months, 6 months, and 9 months after injection with Crespine ${ }^{\circledR}$ Gel. Two main questions were answered to evaluate the amount of knee pain experienced during daily activities: the level of pain for each knee and the degree of difficulty in performing daily physical activities. The analysis of the primary efficacy endpoint showed that the WOMAC pain score was significantly reduced by intra-articular Crespine $^{\circledR}$ Gel administration after 9 months $(P<0.0001$; Table 2 and Figure 3).

\section{Statistical analysis of the efficacy of secondary endpoints}

The results of the analysis of the stiffness scores obtained from the WOMAC questionnaire are presented in Table 3 and Figure 4. The statistical analysis results for the physical function scores obtained from the WOMAC questionnaire are presented in Table 4 and Figure 5.

\section{OMERACT-Osteoarthritis Research Society International (OARSI) responders}

The participants who showed improvement in at least one category in the WOMAC pain assessment were classified as responders. The numbers of $20 \%$ responders, $50 \%$ responders, and 70\% responders were reported. Most participants 


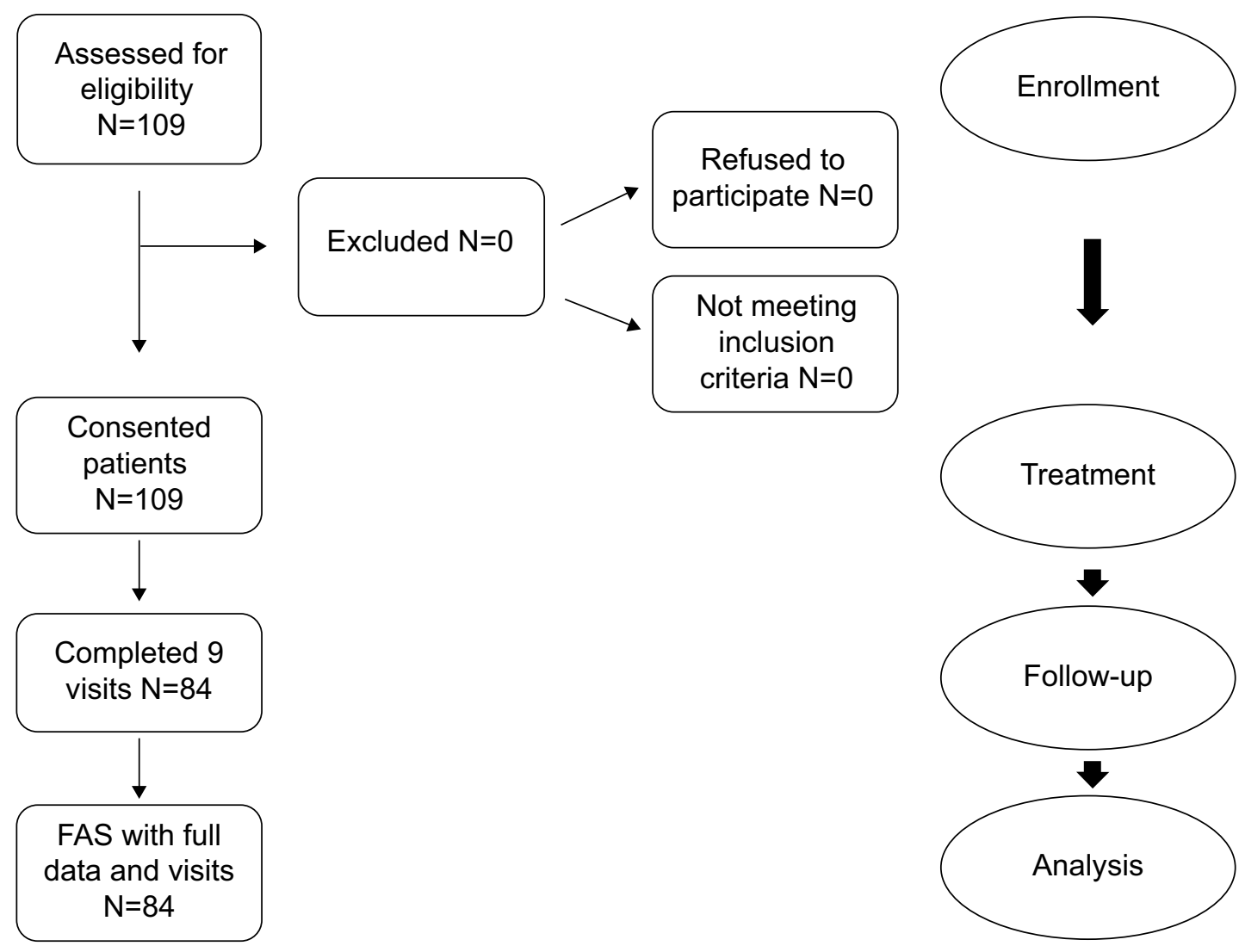

Figure I Participant flow at different study phases.

Abbreviation: FAS, full analysis set.

$(83,99 \%)$ showed $20 \%$ improvement and were considered to be $20 \%$ responders, while $82 \%$ of participants ( 69 patients) showed $50 \%$ improvement or better, while $75 \%$ of participants (63 patients) were considered $70 \%$ responders. These

Table I Demographic variables and disease status at baseline

\begin{tabular}{ll}
\hline Baseline measure & Mean (SD) or $\%$ \\
\hline Age (years) & $55.83(9.34)$ \\
Sex & \\
$\quad$ Female & $36.36 \%$ \\
$\quad$ Male & $63.64 \%$ \\
Weight (kg) & $84.84(12.39)$ \\
Height (m) & $1.67(0.10)$ \\
Body mass index (BMI) kg/m ${ }^{2}$ & $30.50(4.90)$ \\
Ethnicity & \\
Middle Eastern & \\
$\quad$ Asian & $100 \%$ \\
$\quad$ Caucasian & \\
Right knee pain & $93.90 \%$ \\
Left knee pain & $96.22 \%$ \\
Radiology (X-ray) grading & \\
Grade I & $8.33 \%$ \\
Grade II & $34.52 \%$ \\
Grade III & $55.95 \%$ \\
Grade IV & $1.19 \%$ \\
\hline
\end{tabular}

results confirmed the efficacy of intra-articular injection of Crespine ${ }^{\circledR} \mathrm{Gel}$ on OA.

\section{Safety assessment and adverse events}

The safety evaluation was performed 72 hours after analyzing the questionnaire, in order to determine whether the pain in the knee receiving the Crespine ${ }^{\circledR}$ Gel injection had increased. Redness and swelling, in addition to pain at the site of injection, were observed. Thirty three of the 84 participants in the FAS suffered from increased pain 72 hours after receiving the Crespine ${ }^{\circledR}$ Gel injection (ie, $39 \%$ of participants completing the study and the follow-up visits had pain due to the injection). Of the 33 participants with increased pain after the Crespine ${ }^{\circledR}$ Gel injection, 17 participants suffered from pain alone, seven participants suffered from pain and redness of the knee joint, five participants suffered from pain and swelling, and four participants suffered from simultaneous pain, swelling, and redness. All 33 patients with increased pain after the Crespine ${ }^{\circledR}$ Gel injection had limited mobility of the knee joint at 72 hours post-injection. Most participants suffering from the aforementioned side effects were treated with pain medications, including paracetamol 


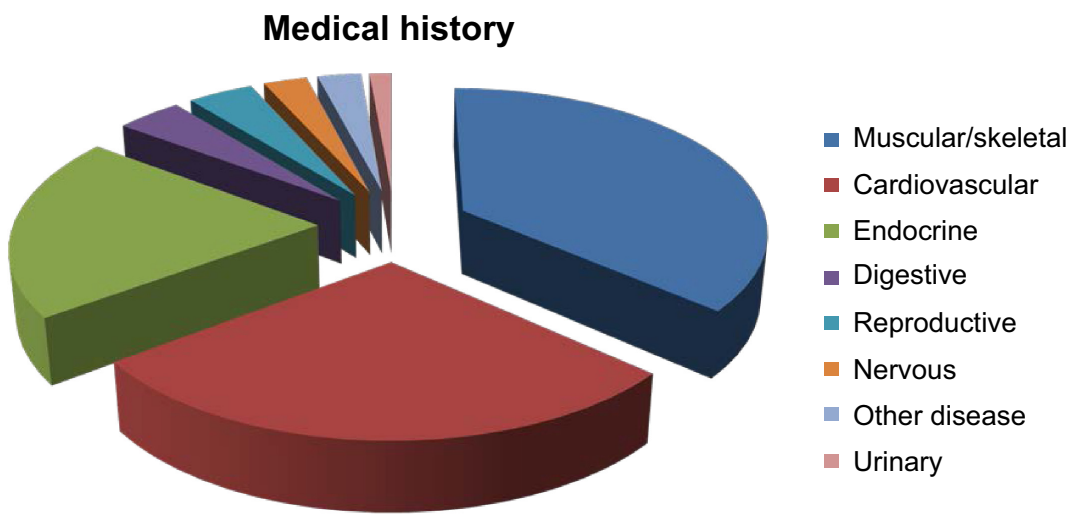

Figure 2 Medical history of enrolled participants.

Table 2 Effect of Crespine ${ }^{\circledR}$ Gel on the primary study endpoint (OA knee pain)

\begin{tabular}{lllll}
\hline Visit & Time (months) & Mean pain score & SD & t-value \\
\hline Baseline & 0 & 10.988 & 2.219 & - \\
1 & 1 & 4.786 & 3.802 & 12.91 \\
2 & 2 & 3.726 & 3.472 & 16.15 \\
3 & 3 & 3.119 & 2.834 & 20.03 \\
4 & 4 & 2.833 & 2.652 & 21.61 \\
5 & 5 & 2.607 & 2.479 & 23.08 \\
6 & 6 & 2.869 & 3.025 & 19.83 \\
7 & 7 & 3.036 & 3.141 & 18.95 \\
8 & 8 & 3.286 & 3.435 & 17.26 \\
9 & 9 & 3.357 & 3.549 & 16.71 \\
\hline
\end{tabular}

Notes: The two-sided $t$-tests were performed using a $95 \%$ confidence interval $(95 \% \mathrm{Cl}), P<0.000 \mathrm{I}$. All tests were performed and compared with baseline measurements. Abbreviation: OA, osteoarthritis.

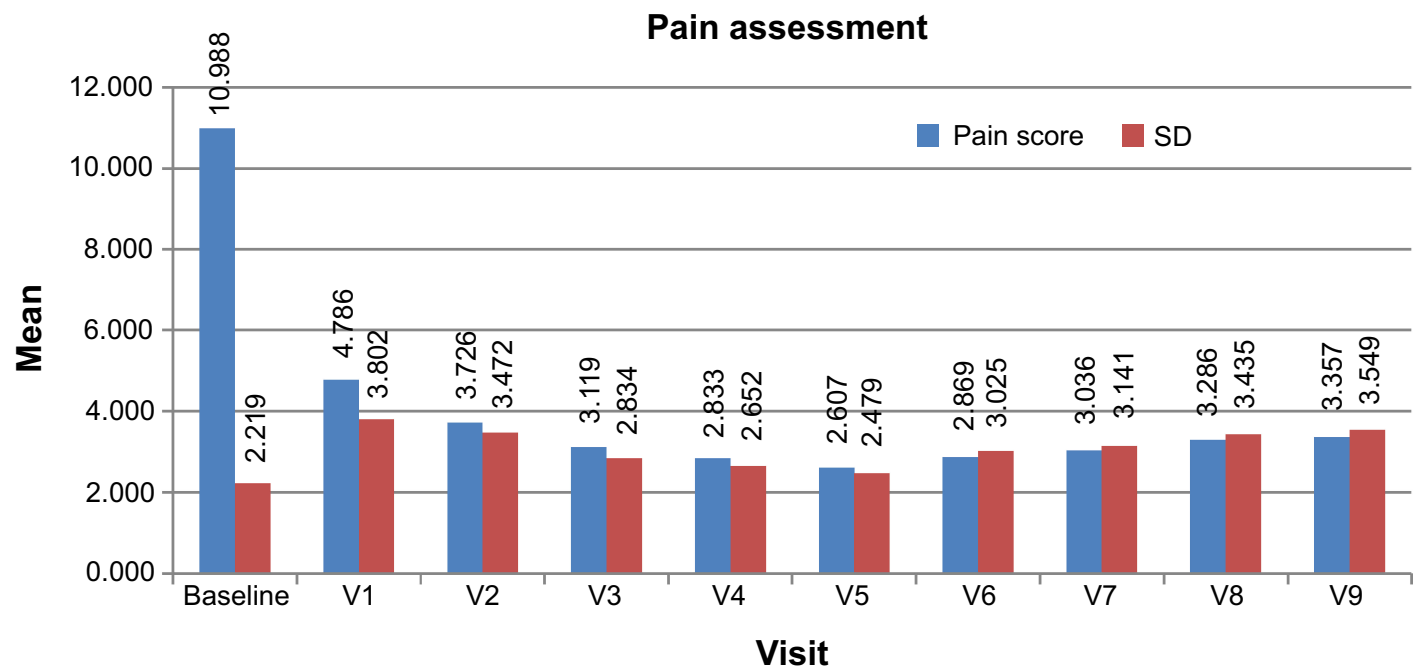

Figure 3 Improvement in pain experienced by the enrolled participants as illustrated by the reduction in the mean WOMAC pain score throughout the follow-up visits. The reduction in the WOMAC pain score as a function of time is also illustrated.

Abbreviation: WOMAC, Western Ontario and McMaster Universities Arthritis Index. 
Table 3 Effect of Crespine ${ }^{\circledR}$ Gel on the WOMAC stiffness score

\begin{tabular}{lllll}
\hline Visit & Time (months) & Mean stiffness score & SD & t-value \\
\hline Baseline & 0 & 3.774 & 1.966 & - \\
1 & 1 & 1.048 & 1.536 & 10.02 \\
2 & 2 & 0.524 & 0.925 & 13.71 \\
3 & 3 & 0.441 & 0.827 & 14.33 \\
4 & 4 & 0.345 & 0.784 & 14.85 \\
5 & 5 & 0.333 & 0.734 & 15.03 \\
6 & 6 & 0.452 & 1.113 & 13.48 \\
7 & 7 & 0.357 & 0.801 & 14.75 \\
8 & 8 & 0.321 & 0.809 & 14.89 \\
9 & 9 & 0.417 & 1.099 & 13.66 \\
\hline
\end{tabular}

Notes: The two-sided $t$-tests were performed using a $95 \%$ confidence interval $(95 \% \mathrm{Cl}), P<0.0001$. All tests were performed and compared with baseline measurements. Abbreviation: WOMAC, Western Ontario and McMaster Universities Arthritis Index.

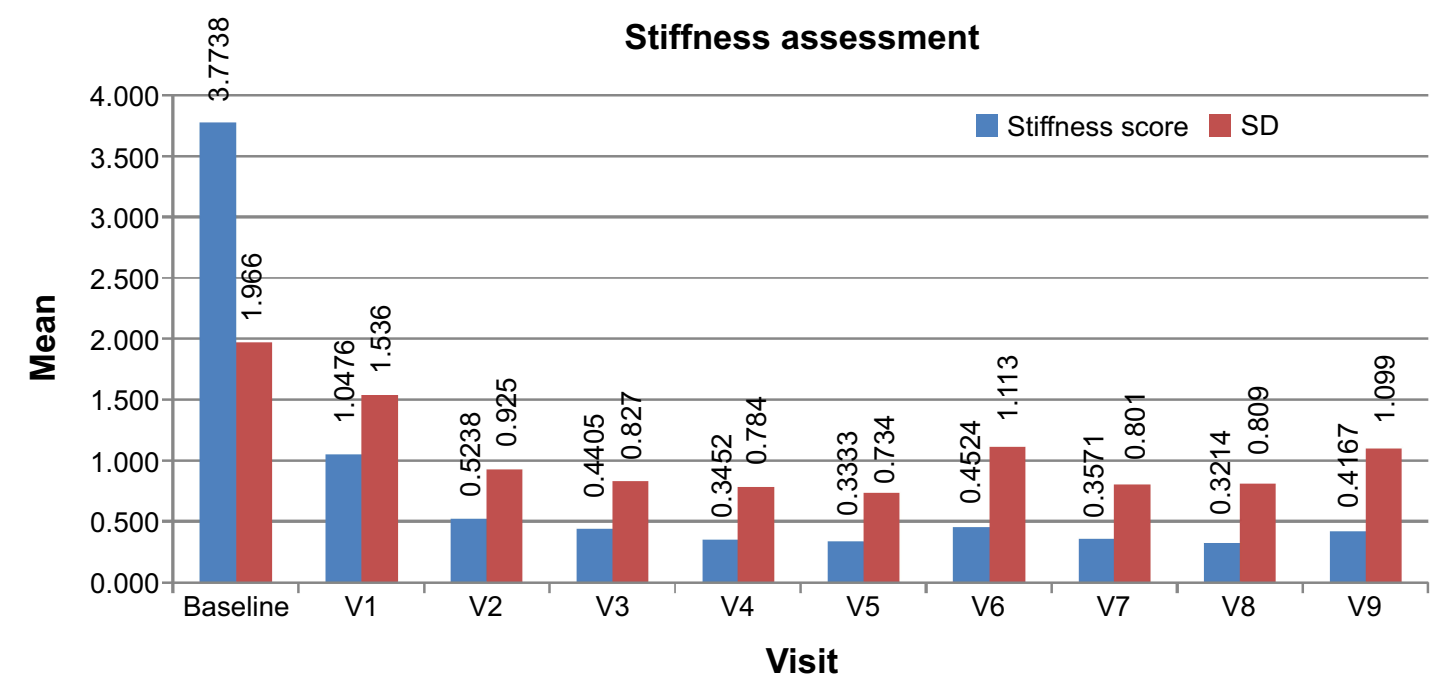

Figure 4 Reduction in the WOMAC joint stiffness score after Crespine ${ }^{\circledR}$ Gel injection. After treatment, the WOMAC joint stiffness score decreased as a function of time, indicating improvement in knee function after Crespine ${ }^{\circledR}$ Gel injection.

Abbreviation: WOMAC, Western Ontario and McMaster Universities Arthritis Index.

Table 4 Effect of Crespine ${ }^{\circledR}$ Gel on the WOMAC physical function score

\begin{tabular}{lllll}
\hline Visit & Time (months) & $\begin{array}{l}\text { Mean physical } \\
\text { function score }\end{array}$ & SD \\
\hline Baseline & 0 & 34.345 & 9.317 & - \\
1 & 1 & 16.190 & 12.266 & 10.007 \\
2 & 2 & $13.57 \mid$ & 12.046 & 12.50 \\
3 & 3 & 11.988 & 10.876 & 14.31 \\
4 & 4 & 10.167 & 9.329 & 16.81 \\
5 & 5 & 9.929 & 9.442 & 16.87 \\
6 & 6 & 10.488 & 10.555 & 15.53 \\
7 & 7 & 10.452 & 10.295 & 15.77 \\
8 & 8 & 11.429 & 10.904 & 14.64 \\
9 & 9 & 11.488 & 11.423 & 14.21 \\
\hline
\end{tabular}

Notes: The two-sided $t$-tests were performed using a $95 \%$ confidence interval $(95 \% \mathrm{Cl}), P<0.000 \mathrm{I}$. All tests were performed and compared with baseline measurements. The values in bold show the peak improvement post-injection.

Abbreviation: WOMAC, Western Ontario and McMaster Universities Arthritis Index. 


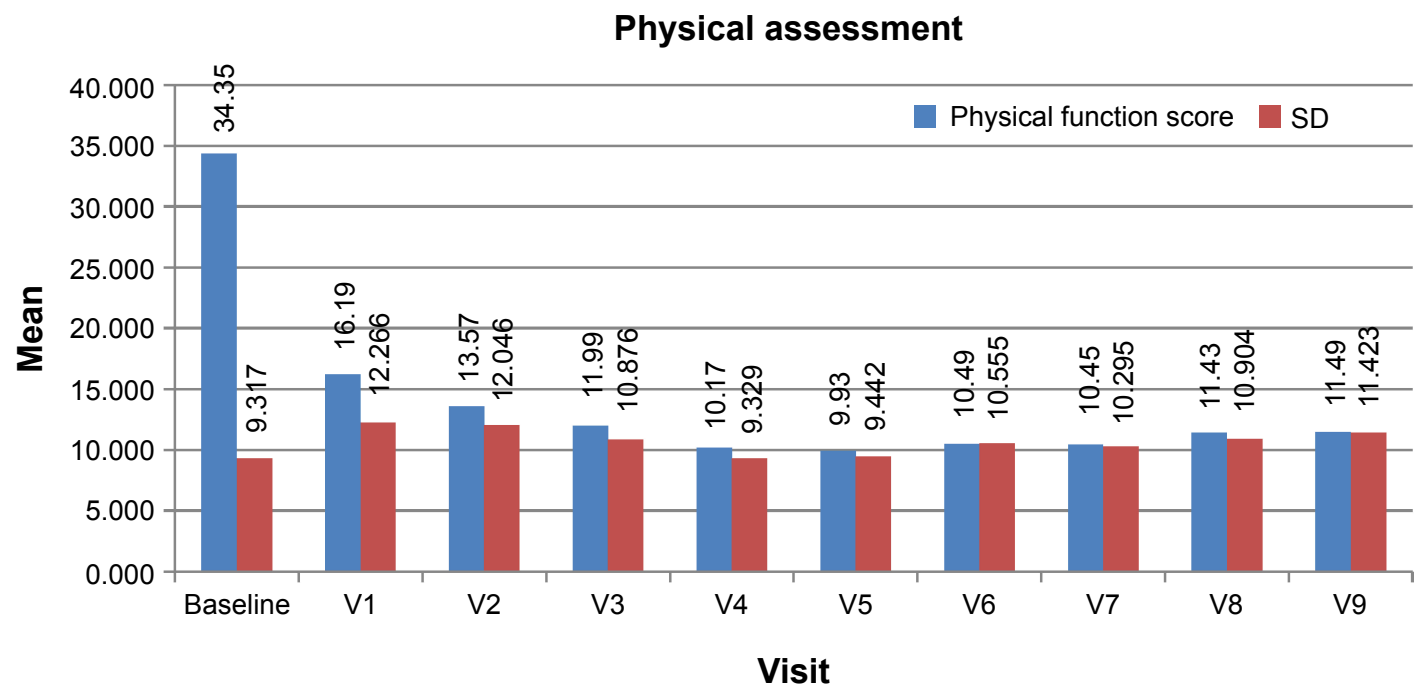

Figure 5 Improvement in the physical function score as a function of time after Crespine ${ }^{\circledast}$ Gel injection.

(500 mg tablets), diclofenac tablets, and mefenamic acid tablets. The pain caused by the injection improved after about 2-3 days in patients taking pain medications, while this pain persisted slightly longer in patients who did not receive any pain medications. Four participants suffered from limited mobility of the knee after receiving the Crespine ${ }^{\circledR}$ Gel injection without experiencing pain, redness, or swelling. Of these 4 participants, three took paracetamol ( $500 \mathrm{mg}$ tablets), while one patient took diclofenac potassium tablets, and all four participants improved in about 2-3 days.

These results show that Crespine ${ }^{\circledR}$ Gel injections have an excellent safety and tolerability profile with no serious side effects. The side effects associated with Crespine ${ }^{\circledR}$ Gel injections were primarily local, such as transient pain, redness, and swelling at the injection site. The local side effects that occurred during the study as a result of Crespine ${ }^{\circledR}$ Gel injections resolved spontaneously or with symptomatic treatment of pain and swelling.

\section{Discussion}

Current treatment approaches, such as those developed by the ACR and the OARSI, position HA as a secondary treatment following nonpharmacological therapies for people who are unresponsive to acetaminophen or NSAIDs, or who cannot take these medications. However, there is evidence to support the use of HAs earlier in the OA treatment paradigm. Patients with lower grades of OA reported a significantly better or much better response to hylan G-F 20 than patients with higher grades of OA. Patients who have been diagnosed with OA within a year are more likely to benefit from hylan G-F 20 than those who have been diagnosed for longer than a year. It has also been demonstrated that patients with less severe OA who received an HA product required fewer analgesics and were less likely to need assistive devices. Collectively, these findings suggest that using HA sooner after OA diagnosis could benefit patients. ${ }^{11,12}$

In addition, HAs are at least as effective as NSAIDs, if not more effective, and have been shown to be safer than NSAIDs and COX-2 inhibitors. HAs are not associated with the potentially serious gastrointestinal problems of NSAIDs or the cardiovascular safety issues of newer COX-2 inhibitors. Given their demonstrated efficacy and safety, as well as the drawbacks of more conservative therapies, HAs could decrease the need for concomitant NSAIDs and corticosteroids in patients with OA, although hylans and HAs are not currently recommended for concomitant use with any other therapies. ${ }^{11,12}$

\section{Interpretation}

This study demonstrated that HA present in Crespine ${ }^{\circledR} \mathrm{Gel}$ injection improves physical knee function, and reduces pain and stiffness associated with knee OA, while exhibiting an excellent safety profile, especially in comparison with NSAIDs and COX-2 inhibitors. At 1 month post-injection, statistically significant improvements in WOMAC pain, stiffness, and functional performance subscores were noted. The pretreatment subscores for pain, stiffness, and functional performance were eleven (total score of 20), 3.8 (total score of eight), and 34 (total score of 68), respectively, and these scores improved to $4.8,1.0$, and 16.2, respectively after Crespine ${ }^{\circledR}$ Gel administration $(P<0.0001$ for each variable). Peak improvement was noted 5 months after Crespine ${ }^{\circledR}$ Gel administration, when 
the scores for pain, stiffness, and functional performance had decreased to $2.60,0.33$, and 9.90, respectively. Significant improvement was maintained 9 months after the injection, as indicated by a pain score of 3.36, a stiffness score of 0.42 , and a functional performance score of 11.5.

The reductions in the rates of pain, stiffness, and difficulty performing necessary physical functions $(73.8 \%, 90.2 \%$, and $69 \%$ of patients, respectively) were statistically and clinically relevant. Moreover, these changes were associated with reduced rates of difficulty performing social and emotional functions (77.9\% and $84.3 \%$ of patients, respectively). Thus, the efficacy of intra-articular Crespine ${ }^{\circledR}$ Gel injection was high in the FAS of selected participants.

\section{Limitation}

The current study is limited in that it was not a double-blinded controlled trial, and thus the treatment used in the study was not compared to a placebo or to commonly used medications such as NSAIDs. Therefore, the design may have introduced bias into the results. Additionally, the sample size was not calculated statistically, but was instead estimated after the study to have sufficient power to exclude the patients who dropped out of the study, and to provide sufficient evidence that intra-articular Crespine ${ }^{\circledR}$ Gel injection is an effective treatment for OA of the knee. Finally, the WOMAC clinical questionnaire does not distinguish one knee from the other when the patient has bilateral knee OA. Therefore, in cases where both knees are affected, the knee selected for treatment was the one considered "worse" by the patient.

\section{Conclusion}

Our results show that HA should be encouraged as an alternative to oral analgesics and considered as an adjunct treatment, because it can decrease the required dose of oral analgesics and delay potential future surgical intervention. Therefore, HA viscosupplementation is a valuable treatment option for $\mathrm{OA}$ of the knee. By demonstrating the efficacy and safety of intra-articular Crespine ${ }^{\circledR}$ Gel administration, the results reported here provide a rationale for the use of Crespine ${ }^{\circledR}$ Gel as a treatment for patients with OA of the knee.

Future randomized trials should be carried out with larger sample sizes to identify optimal treatment alternatives for patients with OA of the knee. Furthermore, studies should be conducted to evaluate whether there are synergistic effects of combining an HA product with current products used for treating patients with $\mathrm{OA}$, as well as to determine the effect of intra-articular Crespine ${ }^{\circledR}$ Gel injections on patients with different grades of knee OA.

\section{Acknowledgments}

Clinical Quest (a clinical research center) prepared the study protocol and handled the data, statistics, and initial study report (Tel +962 6461 0014; Fax +962 6461 6605). Biopolymer GmbH \& Co., KG (Germany) funded the study without any role in the study or publication. The Jordanian Food and Drug Administration registered and approved the study under reg. number MDV-JO-201/01.

\section{Author contributions}

All authors declare their contribution to the conception and design of the study, critical revision of the article for intellectual content, and final approval of the study report to be submitted. Khaldoon Bashaireh, acted as the principal investigator and contributed to the analysis and interpretation of data. Rami N Al-Khateeb contributed to the analysis and interpretation of data and the preparation of the final report to be submitted, and is responsible for the integrity of the work as a whole, from inception to finished article.

\section{Disclosure}

The authors report no conflicts of interest in this work. The corresponding author declares that a business relationship exists with the funding organization in addition to his authorship role.

\section{References}

1. Berg P, Olsson U. Intra-articular injection of non-animal stabilised hyaluronic acid (NASHA) for osteoarthritis of the hip: a pilot study. Clin Exp Rheumatol. 2004;22(3):300-306.

2. Altman RD, Akermark C, Beaulieu AD, Schnitzer T; Durolane International Study Group. Efficacy and safety of a single intra-articular injection of non-animal stabilized hyaluronic acid (NASHA) in patients with osteoarthritis of the knee. Osteoarthritis Cartilage. 2004;12(8): 642-649

3. Salaffi F, Leardini G, Canesi B, et al. Reliability and validity of the Western Ontario and McMaster Universities (WOMAC) osteoarthritis index in Italian patients with osteoarthritis of the knee. Osteoarthritis Cartilage. 2003;11(8):551-560.

4. Pagnano M, Westrich G. Successful nonoperative management of chronic osteoarthritis pain of the knee: safety and efficacy of retreatment with intra-articular hyaluronans. Osteoarthritis Cartilage. 2005;13(9): 751-761.

5. Aggarwal A, Sempowski IP. Hyaluronic acid injections for knee osteoarthritis. Systematic review of the literature. Can Fam Physician. 2004:50:249-256

6. Tasciotaoglu F, Oner C. Efficacy of intra-articular sodium hyaluronate in the treatment of knee osteoarthritis. Clin Rheumatol. 2003;22(2): $112-117$.

7. Fraser JR, Laurent TC, Laurent UB. Hyaluronan: its nature, distribution, functions and turnover. $J$ Intern Med. 1997;242(1):27-33.

8. Jüni P, Reichenbach S, Trelle S, et al; Swiss Viscosupplementation Trial Group. Efficacy and safety of intraarticular hylan or hyaluronic acids for osteoarthritis of the knee: a randomized controlled trial. Arthritis Rheum. 2007;56(11):3610-3619. 
9. Roque V, Agre M, Barroso J, Brito I. Managing knee ostheoarthritis: efficacy of hyaluronic acid injections. Acta Reumatol Port. 2013;38(3): 154-161.

10. Bellamy N, Bell MJ, Goldsmith CH, et al. Evaluation of WOMAC 20, 50,70 response criteria in patients treated with hylan G-F 20 for knee osteoarthritis. Ann Rheum Dis. 2005;64(6):881-885.
11. Reid MC. Viscosupplementation for osteoarthritis: a primer for primary care physicians. Adv Ther. 2013;30(11):967-986.

12. Palmieri B, Rottigni V, Iannitti T. Preliminary study of highly crosslinked hyaluronic acid-based combination therapy for management of knee osteoarthritis-related pain. Drug Des Devel Ther. 2013;1(7): $7-12$.

\section{Publish your work in this journal}

Drug Design, Development and Therapy is an international, peerreviewed open-access journal that spans the spectrum of drug design and development through to clinical applications. Clinical outcomes, patient safety, and programs for the development and effective, safe, and sustained use of medicines are a feature of the journal, which

has also been accepted for indexing on PubMed Central. The manuscript management system is completely online and includes a very quick and fair peer-review system, which is all easy to use. Visit http://www.dovepress.com/testimonials.php to read real quotes from published authors.

Submit your manuscript here: http://www.dovepress.com/drug-design-development-and-therapy-journal 